IRA-International Journal of Technology \& Engineering ISSN 2455-4480; Vol.07, Issue 02 (2017)

Pg. no. 10-16

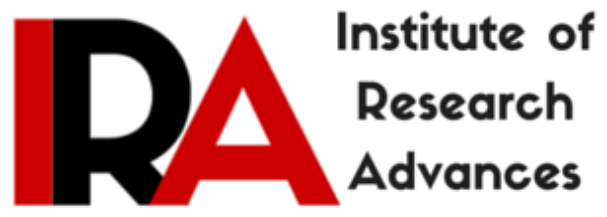

\title{
Biomedical Waste Management in Hospitals - A Review
}

\author{
Deepali Agrawal $^{1}$, Dr. Parag Dalal ${ }^{2}$, Dr. J. K. Srivastava 3 \\ 1. Research Scholar Department of Chemical Engineering, Ujjain Engineering College, \\ Ujjain (M.P.)-456010, India. \\ 2. Asst. Prof. Chemical Engineering Department, Ujjain Engineering College, Ujjain \\ (M.P)-456010, India. \\ 3. Professor and Head Chemical Engineering Department, Ujjain Engineering College, \\ Ujjain (M.P.)-456010, India.
}

Type of Reviewed: Peer Reviewed.

DOI: http://dx.doi.org/10.21013/jte.v7.n2.p1

\section{How to cite this paper:}

Agrawal, D., Dalal, P., \& Srivastava, J. (2017). Biomedical Waste Management in Hospitals A Review. IRA-International Journal of Technology \& Engineering (ISSN 2455-4480), 7(2), 1016. doi:http://dx.doi.org/10.21013/jte.v7.n2.p1

(C) Institute of Research Advances

\section{(cc) EY-NC}

This work is licensed under a Creative Commons Attribution-Non Commercial 4.0 International License subject to proper citation to the publication source of the work.

Disclaimer: The scholarly papers as reviewed and published by the Institute of Research Advances (IRA) are the views and opinions of their respective authors and are not the views or opinions of the IRA. The IRA disclaims of any harm or loss caused due to the published content to any party. 


\section{ABSTRACT}

Biomedical waste management is receiving larger attention because of recent regulations of the biomedical Wastes (Management \&amp; Handling Rules, 1998). Inadequate management of biomedical waste may be associated with risks to healthcare employees, patients, communities and their environment. The current study was conducted to assess the quantities and proportions of different constituents of wastes, their handling, treatment and disposal way in numerous health-cares. In this research, we try to elaborate and discuss the of Bio-medical waste management procedure of Ujjain city. Various health care units were surveyed using a modified survey form for waste management. This form was obtained from the world Health Organization (WHO), with the aim of assessing the processing systems for biomedical waste disposal. Hazards related to poor biomedical waste management and shortcomings in the existing system were identified. The development of waste management policies, plans, and protocols are suggested, in addition to establishing training programs on correct waste management for all healthcare workers.

Keyword- Biomedical waste, Healthcare care unit, WHO, BMW

\section{Introduction}

A biomedical waste is a form which contains infectious waste in it. All hospital waste, health care waste, research waste, laboratory waste includes in it. It can be in any form of solid, liquid or semi-solid. Infectious waste include blood, injection, needle, syringe, body imputed parts, used bandages and dressings, human or animal tissues, sharps, discarded gloves etc.

Biomedical waste becomes fatal because of two principle reasons -

i. first is infectivity

ii. second is toxicity

\section{BMWs consist of -}

i. general waste

ii. Human anatomical waste

iii. discarded drugs and medicines

iv. sharps

v. biotechnological and microbiological waste

vi. animal waste generated from veterinary hospital and during research

vii. solid waste i.e. dressings, bandages, discarded gloves, packing cover, material or cloth contaminated with blood, used cotton, tubes etc.

viii. liquid waste

ix. incinerated waste

Management of BMWs is still in its infancy across all over the world because there is a lot of a problem among generation, operation, treatment, disposal, safe management and lack of knowledge and awareness.

Generally, the BMWs are regulated and management by according to various rules, regulations and standards in different countries. Total biomedical waste generation in country is 484 TPD from 1, 68,869 health care facilities out of which 447 TPD is treated. India generated about three million tons of biomedical waste every year and the amount is expected to grow at $8 \%$ annually. The GOI specifies that hospital waste management is a part of hospital hygiene and maintenance which involves theirs each and every activity begins from generation to disposal.

WHO (world health organization) informs that $85 \%$ of hospital waste are non hazardous, $10 \%$ infectious, $5 \%$ are non-infectious but they are included in hazardous waste. About $15-35 \%$ of hospital waste is 
treated as infectious waste.

Type of waste \& category of waste

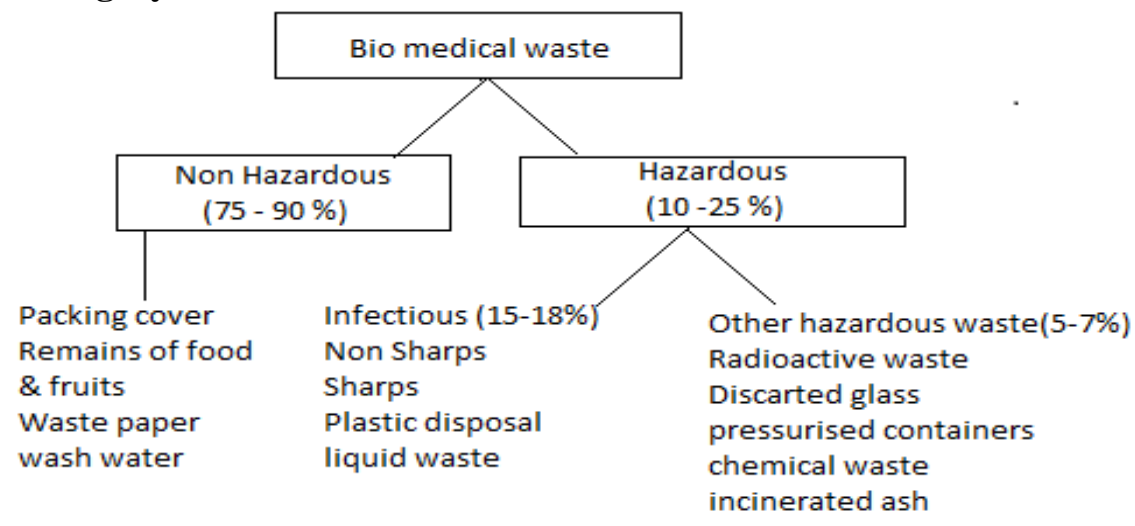

Table 1. Categories of biomedical wastes

Category Source of waste

1 Human Anatomical Waste (human tissues, organs, body parts)

2 Animal Waste (animal tissues, organs, body parts, carcasses, bleeding parts, fluid, blood and experimental animals used in research, waste generated by veterinary hospitals, colleges, discharge from hospitals, animal houses)

3 Microbiology \& Biotechnology Waste (wastes from laboratory cultures, stocks or specimens of micro-organisms live or attenuated vaccines, human and animal cell culture used in research and industrial laboratories, wastes from production of biological, toxins, dishes and devices used for transfer of cultures)

4

Waste Sharps(needles, syringes, scalpels, blades, glass, etc. that may cause puncture and unused sharps)

5

Discarded Medicines \& Cytotoxic drugs (wastes comprising of outdated, contaminated and discarded medicines)

6 Soiled Waste (items contaminated with blood and body fluids including cotton, dressings, soiled plaster casts, lines, beddings, other material contaminated with blood.

7

Solid Waste (wastes generated from disposable items other than waste sharps such as tabbing, catheters, intravenous sets etc.)

8

Liquid Waste(waste generated from laboratory and washing, cleaning, housekeeping and disinfecting activities)

9 Incineration Ash (ash from incineration of any bio-medical waste)

10

Chemical Waste (chemicals used in production of biological, chemicals used in disinfection, as insecticides, etc.)
Treatment and Disposal

Incineration/deep burial

Incineration/deep burial

Local autoclaving /

microwaving incineration

Disinfection (chemical

treatment /autoclaving/

microwaving and mutilation/

shredding"

Incineration/destruction and

drugs disposal in secured

landfills

Incineration autoclaving/

microwaving

Disinfection by chemical treatment autoclaving/ microwaving and mutilation/ shredding"

Disinfection by chemical treatment and discharge into drains

Disposal in municipal landfill Chemical treatment and discharge into drains for liquids and secured landfill for solids. 


\section{Source of waste generation}
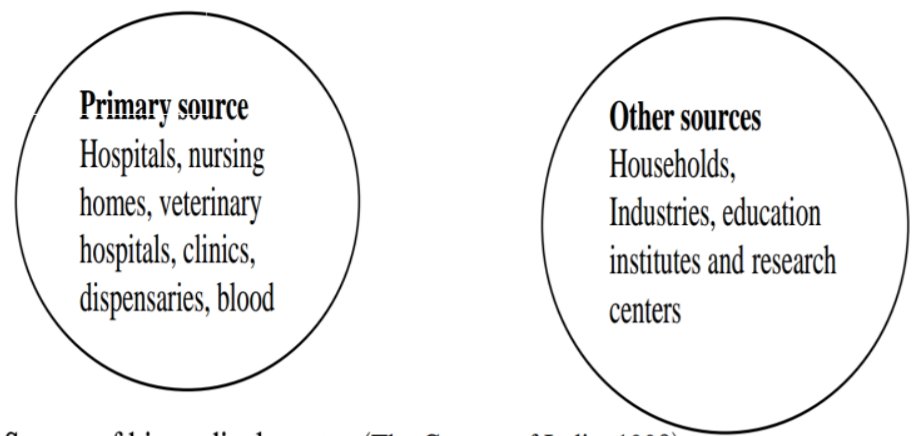

Figure 1. Source of biomedical wastes (The Gazette of India, 1998)

\section{Steps for waste management}

Step 1

Segregation

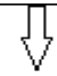

Step 2

Collection and storage

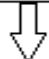

Step 3

Transportation

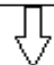

Step 4

Treatment and disposal

\section{Environmental management system for BMW}

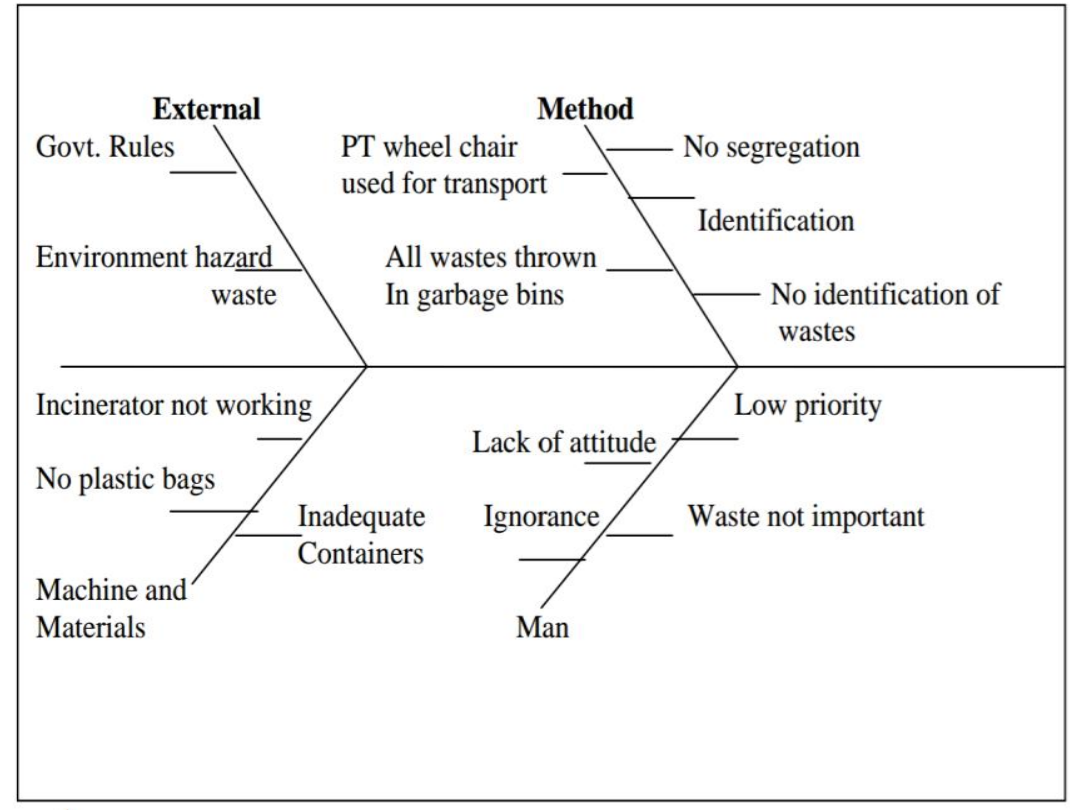




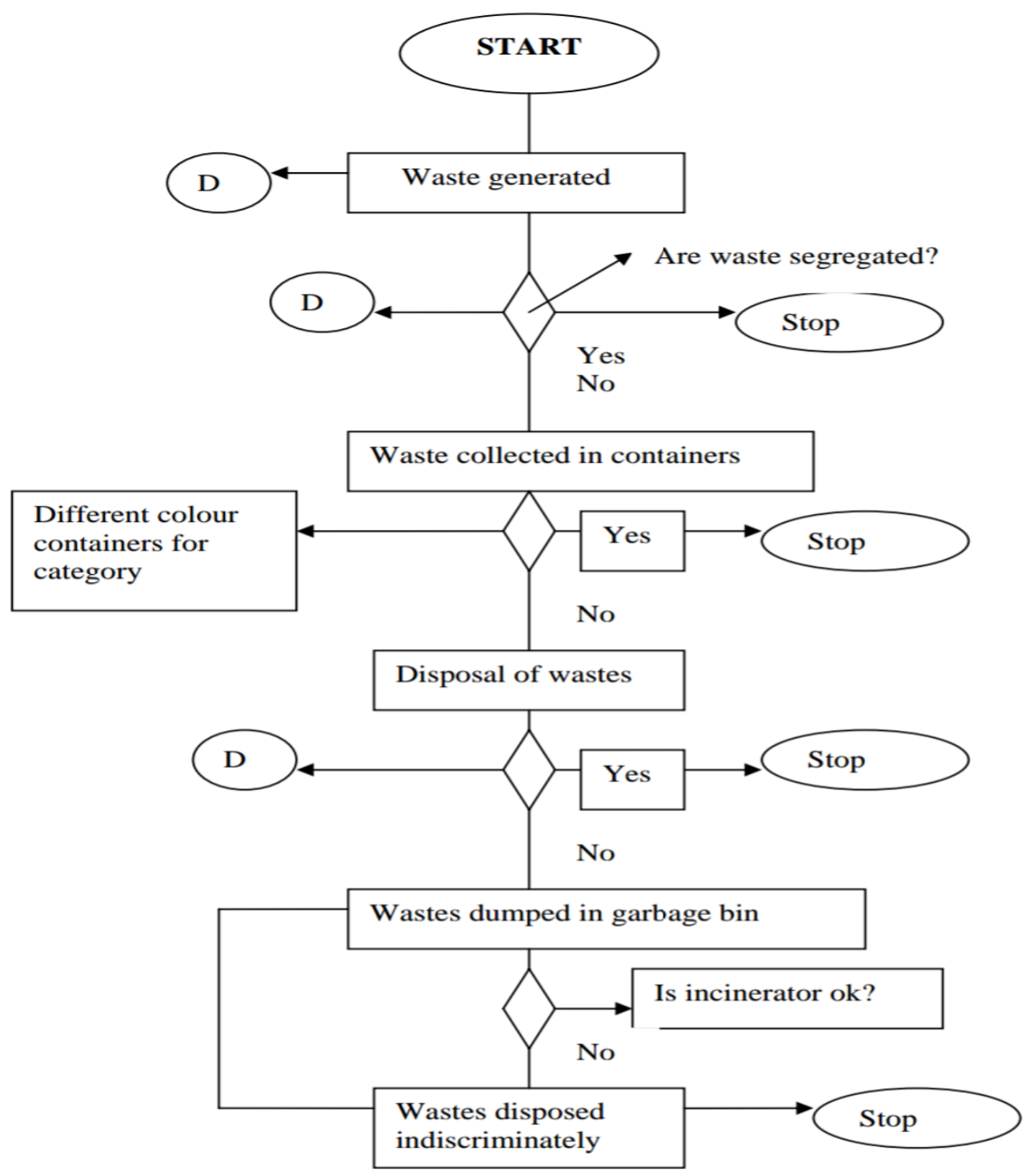

\section{Biomedical waste in Ujjain, India}

With increasing awareness of waste treatment and Swachh Bharat mission, Ujjain city also develop the following procedure for health care waste management for city. Ujjain is generating approximately 225 tons per day of waste out of which about 80 tons are Biomedical Waste. The Government hospitals and some big private hospitals have their own arrangement for treatment of biomedical waste (Dalal 2011). 


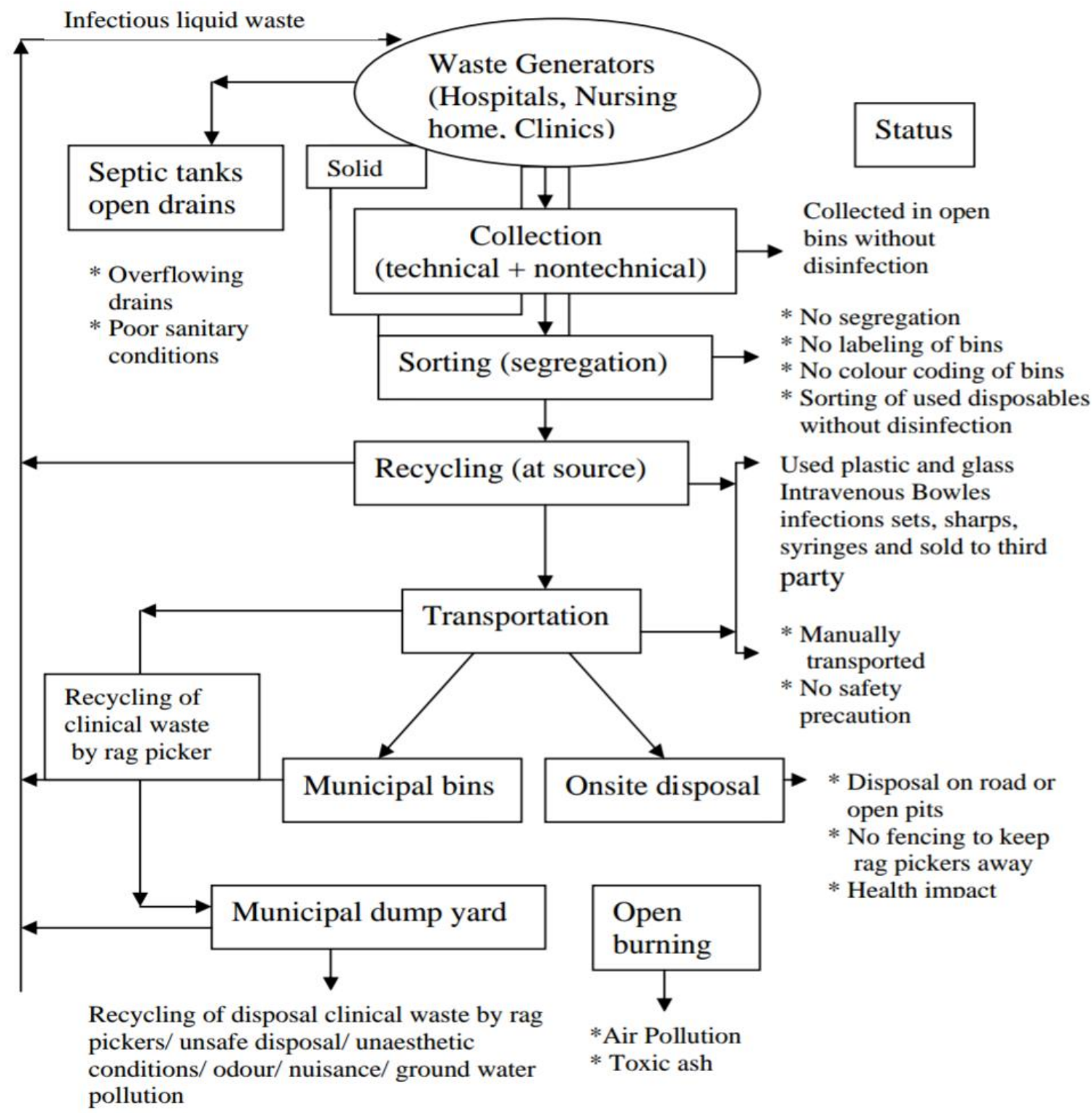

Fig 3. Current Status of medical waste disposal in Ujjain

\section{Problem related to biomedical waste}

-Major issue is lack of knowledge and awareness of importance of waste management among the people. -Main hurdle in health care is the implementation of standards and regulations.

- Carelessness in proper collection and segregation of biomedical waste.

-Inadequate waste maintenance invites other pollution too.

-Unpleasant smell, growth of flies, insects, rodent, and worms may lead to other diseases and injuries too. -Rag pickers and the person who handles the waste is in most danger.

\section{Need of BMW in healthcare centers}

The reason by which proper management is needed in hospital or health cares of Ujjain are as

- If proper management of waste is not done by the organizations then there will be a lot of risk arises due to infection inside as well as at outside of centers. 
- Biomedical waste will cause air, water and soil pollution directly and leads harm to flora and fauna

- Disposable solid and liquids might be repacked and resold in market by illegal traders or unscrupulous elements.

\section{Recommendation}

- Biomedical waste should not be get mixed with other type of noninfectious waste. If by mistaken this happened then this will have treated as BMW.

- If segregation is done at the point of generation or collection then further treatment will be one step less.

- The containers in which collection or segregation is done, it should have labelled with non-washable and easily visible BMW or Hazardous symbol.

- All plastic bags, glass wares, equipment, machines, containers should be as per BIS standards.

- Untreated anatomical waste (humans and animals), solid and biotechnological waste should not be kept more than 48 hours. But if it stored more time then prescribed, then handler should take appropriate steps so that this waste will not affect the surroundings.

- Hand washing and proper drinking water facility must be available at all necessary areas.

- Health care organization should take all the responsibility for maintenance and proper management of their generated waste.

- Patient care area and storage area should be clean on regularly basis.

- Time to time proper collection is needed by responsible persons.

- Waste collection bag should be removed when 3/4 is full.

- Staff handling waste must use PPE and proper rules \& regulations for waste treatment.

- Proper training and guidance should be recommended.

- Waste should be segregated into colour coded bags.

- Waste collection bag should be tied up tightly and transferred to storage place carefully without any spillage.

- At the end of the day, whole waste of each floor is transported or collected to the central collection area and a proper record of each day collection should be maintained.

\section{Reference}

1. http://www.ujjain.nic.in

2. Pollution Control Board, Ujjain

3. Municipal Corporation, Ujjain

4. National guidelines on Hospital waste management. Biomedical waste regulations. 1998.

5. Govt. of India, "Bio-medical waste (management and handling) rules". The gazette of India. Ministry of Environment and Forest. 1998

6. Dalal P. Liquid Bio-Medical Waste Management strategy Environment Conservation Journal Vol. 12(1\&2) 2011 Pg. 87-93 